in order to support novel research into dementia aetiology and course. Methods: Our objective was to present estimates of the prevalence and incidence of dementia cases across EHRs available through EMIF from six sites in five European countries over a nine-year observation period. All six EHR databases (three primary care, three secondary care) initiated data collection either on or before 2002. Diagnosed dementia was ascertained by consensus harmonization of the various clinical/diagnostic coding systems employed. The data sources were described in terms of numbers of dementia cases and person years of EHR data before and after the first diagnosis. Annual incidences and period prevalences of dementia by age and gender were calculated for each database. Results: The total number of cases across the six databases from 2004-2012 was 138,625, and the data coverage for people aged 50 years and over during this period was $38,000,000$ person years.There was reasonable consistency across databases in age-specific prevalence and incidence of cases, both of which were around 30$50 \%$ lower than published estimates from surveys or cohort studies carried out in community samples. Age-associated increases in prevalence and incidence were comparable to those seen in community samples, although with a leveling off in oldest age groups. There were modest increases in prevalences of dementia diagnosis across the nine years analysed but stable incidence rates. Female:male ratios of prevalence and incidence were stable over time. A modest female excess was evident in older age groups for dementia diagnosis prevalence but not for incidence. Conclusions: The EMIF initiative has brought together EHR data on unprecedented numbers of people with dementia. This creates potentially transformative opportunities for novel research, although it is important that study designs are tailored to take into account the recognised under-diagnosis of dementia, as well as the generic strengths, limitations and applicability of EHR data resources.
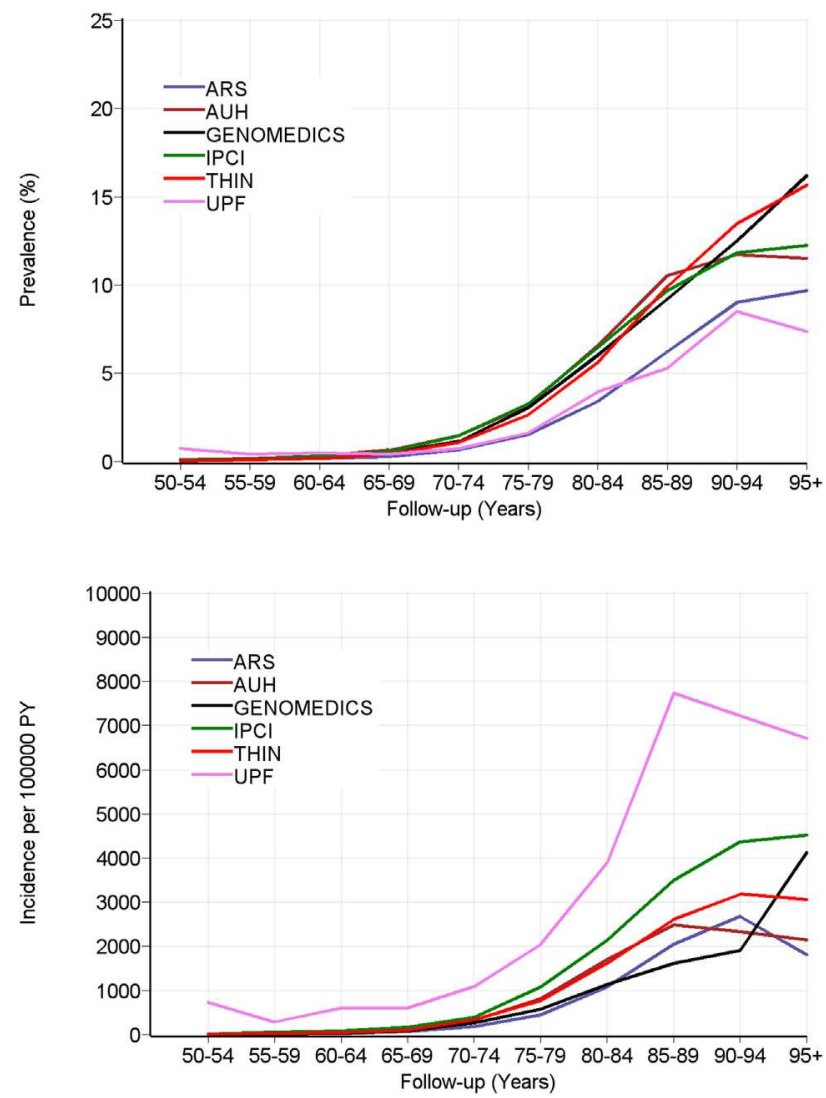

\section{O2-05-03 BIRTH COHORT EFFECTS IN MEMORY FUNCTION AND PRACTICE EFFECTS FROM EPIDEMIOLOGICAL STUDIES OF COGNITIVE IMPAIRMENT AND DEMENTIA}

Hiroko H. Dodge ${ }^{1,2,3}$, Jian Zhu ${ }^{4}$, Tiffany F. Hughes ${ }^{5}$, Beth E. Snitz ${ }^{6}$, Chung-Chou H. Chang ${ }^{7}$, Mary Ganguli ${ }^{7,8},{ }^{1}$ Oregon Health \& Science University, Portland, OR, USA; ${ }^{2}$ University of Michigan, Ann Arbor, MI, USA; ${ }^{3}$ Oregon Center for Aging \& Technology (ORCATECH), Portland, OR, USA; ${ }^{4}$ University of Michigan School of Public Health, Ann Arbor, MI, USA; ${ }^{5}$ Youngstown State University, Youngstown, OH, USA; ${ }^{6}$ University of Pittsburgh, Pittsburgh, PA, USA; ${ }^{7}$ University of Pittsburgh Graduate School of Public Health, Pittsburgh, PA, USA; ${ }^{8}$ University of Pittsburgh School of Medicine, Pittsburgh, PA, USA. Contact e-mail: snitzbe@upmc.edu

Background:Growing evidence suggests the age-specific prevalence and incidence of dementia and cognitive impairment may be stable or even declining over time in the US and other developed countries. Trends in cognitive test performance across birth cohorts may shed light on these effects. Higher educational attainment among the more recently born cohorts is often cited as one of the potential reasons of this trend. Whether age-associated trajectories of memory functions differ by birth cohorts and whether differences can be explained by increasing educational attainment remain unclear. Methods: Pooling data from two prospective population-based studies in two contiguous areas in Pennsylvania between 1987 and 2015, we identified four birth cohorts born 1902-1911, 1912-1921, 1922-1931, and 1932-1943. We first harmonized cognitive test scores across the studies since different memory tests (the Consortium to Establish a Registry for Alzheimer's Disease (CERAD) and the Wechsler Memory Scale-Revised (WMS-R)) were administered. Next we compared age-associated trajectories of memory function and the magnitude of practice effects among the birth cohorts. Using mixed effects models, we first included cohorts, cohort $\mathrm{x}$ time (i.e., age) interaction terms, practice effects and cohort X practice interaction terms, and then added education effects to examine whether education explained the cohort difference in memory trajectories and practice effects. Results: We observed significant cohort effects in learning (immediate recall scores) with its age-associated trajectories showing less steep declines among the later born cohorts in comparison with the earlier born cohorts, independent of education. We also observed cohort effects in practice effects with the higher levels in the later born cohorts, both in learning and memory (delayed recall scores). Conclusions: We found that the more recently born cohorts are experiencing both lesser declines in age associated trajectories in learning, and also larger practice effects in learning and memory. These differences remained after factoring in educational attainment. The finding is consistent with our earlier finding for other cognitive domains where we showed improved age-associated trajectories among recently born cohorts, independent of education. Secular trends other than education could play roles in recently found trends in improved cognitive health in developed countries.

\section{O2-05-04 RESILIENCE FACTORS FOR AMYLOID DEPOSITION AND NEURODEGENERATION ARE DIFFERENT}

Prashanthi Vemuri ${ }^{1}$, Timothy G. Lesnick ${ }^{1}$, Scott A. Przybelski ${ }^{1}$, Rosebud O. Roberts ${ }^{1}$, David S. Knopman ${ }^{1}$, Val J. Lowe ${ }^{1}$, Mary M. Machulda ${ }^{1}$, Michelle M. Mielke ${ }^{1}$, Yonas E. Geda ${ }^{2}$, Ronald C. Petersen ${ }^{1}$, Clifford R. Jack, Jr, ${ }^{1},{ }^{1}$ Mayo Clinic, Rochester, MN, USA; ${ }^{2}$ Mayo Clinic, Scottsdale, AZ, USA. Contact e-mail: Vemuri. Prashanthi@mayo.edu

Background: Important pathological processes (amyloid deposition and neurodegeneration) in Alzheimer's disease (AD) can 